\title{
A Survey on Common Practice in Designing Audio in the User Interface
}

\author{
Christopher Frauenberger, Tony Stockman, Marie-Luce Bourguet \\ Interaction Media Communication, Department of Computer Science \\ Queen Mary, University of London \\ Mile End Road, London E1 4NS, UK \\ \{frauenberger, tonys, mlb\} @dcs.qmul.ac.uk
}

\begin{abstract}
The current practice of designing the auditory mode in the user interface is poorly understood. In this survey, we aim at revealing the common understanding of the role of audio in human-computer interaction and how designers approach design tasks involving audio. We investigate which guidelines and principles participants use in their designs and which guidance is needed to improve the quality of auditory design. The responses are analysed and interpreted by quantitative and qualitative methods. The 86 participants enabled us to draw a relatively accurate picture of how the field is perceived and helped to identify problems in the design of efficient audio in the user interface. The results of the survey are subsequently developed into requirements for a methodological design framework, with the aim to provide easily accessible guidance for designers to integrate audio in the user interface.
\end{abstract}

\section{Categories and Subject Descriptors}

H.5.2 [Information Interfaces and Presentation (e.g., HCI)]: User Interfaces - auditory (non-speech) feedback, theory and methods.

\section{General Terms}

Design, Human Factors, Languages, Theory.

\section{Keywords}

Auditory display, design practice, design framework, survey.

\section{INTRODUCTION}

Audio, as integrative part in the human-computer interface, has been widely neglected in the past. Compared to the advances in visual interaction, the auditory domain not only lacks a similar level of technological standard, but also provides few methodological means to inform design decisions. This results in audio being used often in an inappropriate and inefficient way, leaving users annoyed, or worse, making the interface inaccessible. Before we endeavour to challenge this problem, however, we need to understand more clearly how designers of human-computer interaction approach auditory design and investigate the performance of existing methodologies in the

(C) Christopher Frauenberger, Tony Stockman, Marie-Luce Bourguet, 2007

Published by the British Computer Society

People and Computers XXI-HCI... but not as we know it:

Proceedings of HCI 2007

Linden J. Ball, M. Angela Sasse, Corina Sas, Thomas C. Ormerod, Alan Dix, Peter Bagnall, and Tom McEwan (Editors) design process. Hence, we conducted the survey presented in this paper.

\subsection{Motivation}

The auditory interaction modality is a powerful and efficient communication channel. Our ears are precise instruments that contribute greatly to the perception of our everyday environments. Having been a neglected area for long, the use of audio in human-technology interaction is now receiving increasing attention. This interest is mainly driven by the increasing need for alternative means of interaction in fields such as mobile, ubiquitous and pervasive computing. The contexts of use of technology have been extended to scenarios in which the classical visual screen raises significant usability issues, e.g. when physical characteristics of devices do not allow appropriate sizes of screens or when users have their eyes occupied with other tasks like navigating through a lively street.

Additionally, restricting communication with technology to a single modality makes it inaccessible for people with certain disabilities. Although most computer systems nowadays incorporate some level of assistive technology for the impaired, access is far from equally efficient and creates another barrier for the integration of the people concerned in their professional and social life alike.

Incorporating efficient auditory artefacts in the user interface will benefit a wide range of users and applications. It is reasonable to predict that it will become even more important with the development of future interaction paradigms.

\subsection{Background}

Despite the fact that research has produced prototypes that have demonstrated the potential of audio (e.g., [1,2]), it has not been propagated into many commercial products. In 1994, Mynatt stated "Current research in auditory interfaces has produced a number of interesting interfaces without resulting in the compilation of design guidelines for creating descendent interfaces"; and she goes on: "This lack of a common design methodology may be partially responsible for the scarcity of commercial interfaces that make use of sound" [3]. Thirteen years later, while the field continues to produce very interesting prototypes, the commercial impact is still marginal. For example, the Apple iPod has a single sound designed to support the interaction: the clicking of the wheel. This leaves it almost inaccessible to all visually impaired users and raises serious issues when interacting in a mobile context. And for all we know about the iPhone it neither incorporates much sound to support interaction.

To make audio a real alternative to visual means in design, it is important to bridge the gap between the design knowledge that 
has been created through research and the real-world design problems. We aim at achieving this by developing a methodological framework to make the design knowledge needed to build efficient auditory interfaces available and accessible in the form of design patterns [4]. However, to be able to develop the methodology around the needs and common practices of HCI designers it is important to have a clear picture of these. The following section will specify in greater detail the research questions that we sought to answer in the light of the overall goal.

\subsection{Research Questions}

In conducting this survey we aimed at answering the following research questions:

- What role does audio play in HCI design?

- What is the attitude towards using audio in user interfaces?

- How would designers approach an audio only design task?

- What guidance for audio design are designers aware of?

- What guidance would they like to see?

By assessing the knowledge of HCI designers and practitioners about incorporating audio in their designs we intend to identify what is missing to enable them to take full advantage of the auditory channel. Through past and current practice, the use of audio has also been linked with a limited range of applications and prejudices about its efficiency and usability dominate the reasoning for using (or excluding) audio. We aim at investigating the stigmata that has branded audio as an interaction paradigm and explore the conception of the scientific field as perceived by designers outside and inside the community.

To accommodate the common practice of designers in the methodological framework it is important to assess how design tasks including audio are approached. Initial stages in the design process are crucial to the outcome; hence, we were interested in finding out how designers approach an audio-only design problem.

Finally, the quantity and the quality of guidance is key to make auditory display design play a bigger role in HCI. Therefore, we probed for guidelines, principles and other forms of guidance and aimed at finding out what designers would like to have.

\subsection{Structure of the Paper}

The remainder of this paper will present the survey and the results. Section 2 introduces the design of the survey including details about the questions and their form of presentation. Section 3 then presents the analysis of the answers given. Different approaches have been taken to interpret the data by quantitative and qualitative methods. Section 4 summarises the results and provides further discussion of the consequences of our research findings. Finally, Section 5 will conclude the paper and lay out the agenda for future work.

\section{SURVEY DESIGN}

To be able to reach a sufficient number of participants the survey was designed as an online questionnaire. It was implemented using the Unit Command Climate Assessment and Survey System ${ }^{1}$, a PHP based survey script running on the departmental server at Queen Mary, University of London. It was advertised by requests for support in mailing-lists of the

\footnotetext{
${ }^{1}$ UCASS http://www.bigredspark.com/survey.html
}

HCI community and communities related to auditory design, namely: chi-web@acm.org, chi-announcements@acm.org (both mailing-lists by ACM/SIGCHI), hcimail@napier.ac.uk (British HCI News), auditory@lists.mcgill.ca (Research in auditory perception) and icad@santafe.org (International Community for Auditory Display mailing list). Additionally, a call for support was published on the Usability News web page ${ }^{2}$.

\subsection{Questions}

Forty questions were distributed across a total of seven webpages with a coherent design, 'back' and 'forward' buttons on each page and a 'finish' button on the last page. The questions were organised in sections with each section targeting a specific aspect.

The first section collected the usual demographical data about participants such as sex, age, profession and education. The following section was probing audio related knowledge and the experience in interaction design. Audio related knowledge was assessed in terms of musical skills and other technical skills such as creating or editing sounds. As for experience, we asked for self-assigned expertise in theoretical and practical user interface design, the modalities used in prior work and a simple classification of target platforms for their designs. If participants had prior experience with using audio in user interfaces they were asked more specifically which type of auditory cues they used and to describe one of their designs in a couple of sentences. Furthermore, we were interested in the motivation behind using audio.

The subsequent section focused on the working environment in which auditory displays are designed. We were interested in the professions of designers who are called in whenever auditory display design is needed and to what extent these designers are integrated in the overall design of a product.

With the following two sections we aimed at collecting information about the design process itself. First, participants were asked about their initial steps in tackling any design problem that was restricted to auditory feedback. Two aspects, the creation of prototypes and the inclusion of the user were specifically highlighted and probed in this context. Second, participants were confronted with a concrete example of an audio-feedback-only design problem. They were asked to describe a design for an Mp3-player employing no screen, but buttons and a joystick to support interaction. The task was to create an auditory display design for navigating the menu that provided access to the content including, in addition to music files, a calendar, contacts and preferences. We asked for initial ideas for solving the problem and encouraged participants to think outside of the box, stressing that technical feasibility was irrelevant. We also asked for the rationale behind their choices.

The last block of questions probed for guidance in the context of auditory display design. First, the awareness of any guidelines or design principles specifically targeting auditory displays was assessed along with their influence on which initial approach was chosen for the Mp3-player design. Subsequently, we asked for guidelines from other areas that participants used in the exercise and how well they adapted to the problem at hand. Finally, we asked for the form of guidance designers would like to have for auditory display design and participants could provide any other thoughts or feedback on the topic.

\footnotetext{
${ }^{2}$ http://www.usabilitynews.com
} 
Thirteen of the questions were designed as free-form text fields, which provoked very different responses. This provided participants with the most flexible way of giving answers, but also left more room for misinterpretation of the questions and of the responses.

\section{RESULTS}

A total of 86 participants completed the survey, which makes it the largest investigation of this sort in the context of auditory display. In this section we present the analysis of the responses using quantitative and qualitative methods.

\subsection{Quantitative Analysis}

The focus of this section lies on data from the survey that could be quantified. For free form text answers a quantitative text analysis was performed assigning tags to answers for occurrences of keywords or their implicit mentioning. It proved to be practical not to define the keywords prior to the coding, but have multiple iterations with keywords emerging from within the data.

\subsubsection{Demographics}

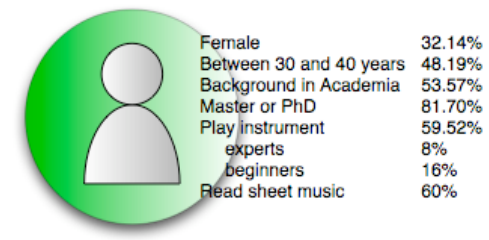

Almost one third (32.14\%) of the participants were female and almost half of all participants (48.19\%) were between 30 and 40 years old. The professional background was balanced with $53.57 \%$ mentioning a background in academia while the other half would be mainly professional HCI practitioners. The level of education was high, as could be expected from the target group. Over $80 \%$ possessed either a Masters or a $\mathrm{PhD}$ as their highest degree $(42.68 \%$ Masters, $39.02 \% \mathrm{PhD})$ with many of the former group being $\mathrm{PhD}$ students. Less than two thirds play a musical instrument $(59.52 \%)$ amongst which the piano was mentioned most often followed by the guitar. Participants assessed their level of playing mostly as average, $8 \%$ were experts and $16 \%$ beginners. About two thirds of all participants said they can read sheet music $(60 \%)$.

\subsubsection{Expertise and Motivation}

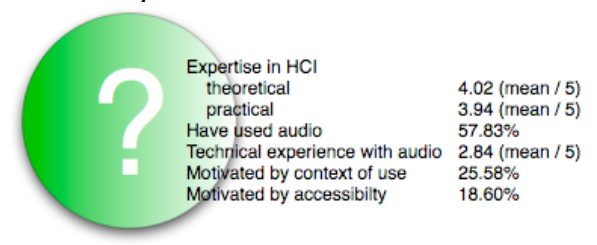

Asked to rate their technical experience with audio (as with creating or editing sounds on the computer) the answers were distributed very evenly between 1 (lowest) and 5 (highest) with a score of 2.84 in average $(\sigma=1.35)$ As expected, the theoretical and practical expertise in designing user interfaces was high. On the same scale, the average level for practical expertise was $3.94(\sigma=1.03)$ and $4.02(\sigma=1.14)$ for theoretical expertise respectively. Almost all $(98.80 \%)$ said they have used the visual interaction channel in their designs, but also over half of the participants have used the auditory channel $(57.83 \%)$. Also, over a quarter mentioned tactile interaction $(26.51 \%)$ and one participant used the olfactory modality $(1.2 \%)$. Most designers reported to develop for the desktop $(83.13 \%)$ and the web

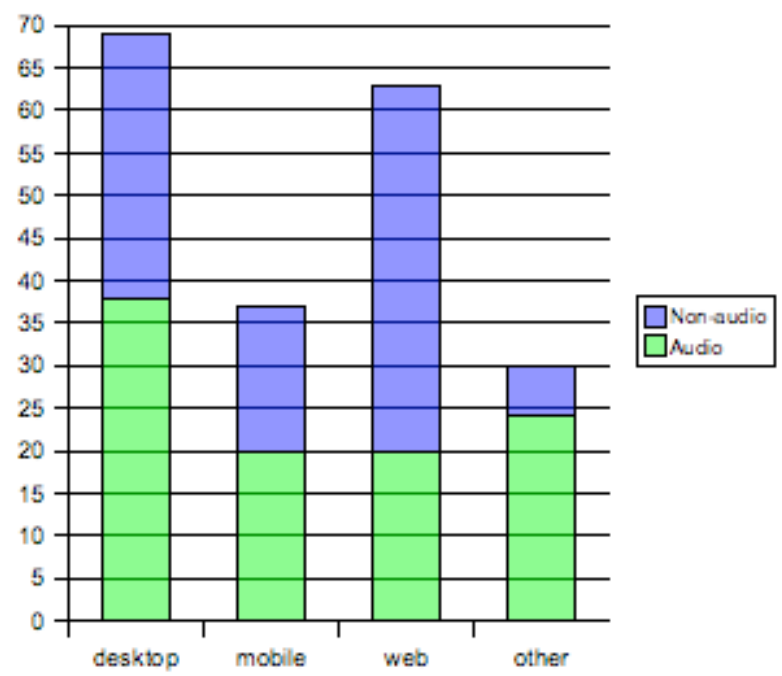

Figure 1. Number of answers for target context for the audio and non-audio group.

(75.90\%). Slightly less than half of all participants also designed in a mobile context (44.58\%).

The majority of the two-thirds of the participants who used audio in their user interfaces used it in less than $20 \%$ of their designs, almost a third $(30.65 \%)$ in less than $10 \%$. However, $14.58 \%$ of those designers said they design exclusively for the auditory domain. User interfaces with audio were also mostly developed for the desktop (64.41\%), but this means almost $20 \%$ less than in the overall numbers probed above. The biggest drop, however, was observed for designs in the context of the web. Only $33.9 \%$ of the designs with audio were targeted to a web-context in comparison to $75.9 \%$ in the overall numbers. The second most answers were given for 'other' contexts $(40.68 \%)$, indicating that many of the designs are tailored towards a very specific context. Figure 1 illustrates the ratios.

When asked to describe one specific application of audio briefly, a 'user interface' was explicitly mentioned in every third description $(30.95 \%)$. Other fields included the perceptualisation of data $(14.29 \%)$, the presentation of multimedia content (14.29\%) and web-content (14.29\%). Also alarms, control room and monitoring type of applications were mentioned. Almost a quarter of the designs described an augmented reality design (23.81\%). Most applications were targeted at improving accessibility (36.84\%), followed by educational designs $(31.58 \%)$, art projects $(26.23 \%)$ and research projects $(21.05 \%)$. Other domains were finance, medical applications and games (2 out of 19 descriptions or $10.53 \%$ each). Within the free-form descriptions, participants mentioned the use of speech most ( 6 times), followed by speech recognition systems ( 3 times), while auditory icons and earcons were mentioned only three times (once and twice respectively). Two-thirds of the participants said that they would use audio to complement other modalities, while $29.51 \%$ said they would mostly use it as an alternative interaction channel. Asked for which kind of audio they would use in their designs, most included 'abstract sounds' (72.41\%), followed by 'speech' $(48.28 \%)$ and 'natural everyday sounds' $(32.76 \%) ; 15.52 \%$ said they would also use 'other' auditory cues.

Over a quarter of the participants who used audio stated that their main motivation for incorporating audio was to accommodate a specific context of use including specific needs of the user $(25.58 \%) ; 18.60 \%$ explicitly mentioned 
accessibility. Another important motivation was to provide a naturalistic experience $(23.36 \%)$ and to match the type of content to be presented with the appropriate human sense $(16.28 \%)$. Also, $16.28 \%$ said that their main motivation is to reinforce information that is also available in other modalities and $11.63 \%$ mentioned the increase of bandwidth for conveying information. Some also stated curiosity as a motivation to use audio ( 3 out of 43 or $6.98 \%$ ).

\subsubsection{Working Environment}

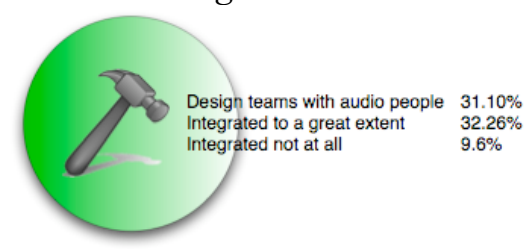

Slightly less than one third of the participants had worked in design teams in which other people were in charge of designing sound for the interface $(31.40 \%)$. Within such design teams, the integration of these colleagues into the overall design was assessed very differently. Although $32.26 \%$ stated that they were integrated to a very great extent, $9.6 \%$ also said they were not integrated at all. The three levels in between received the same number of answers ( 6 out of 31 or 19.35\%). Asked for the professions of the people who designed audio in these teams, all categories were almost checked equally often. 'HCI experts' and 'artists' received the most answers (12 out of 30 or $40 \%$ ), followed by 'sound designer', 'sound engineer' $(36.67 \%$ and $33.33 \%)$ and 'others' $(30.00 \%)$.

\subsubsection{Approach to Audio-Only Designs}

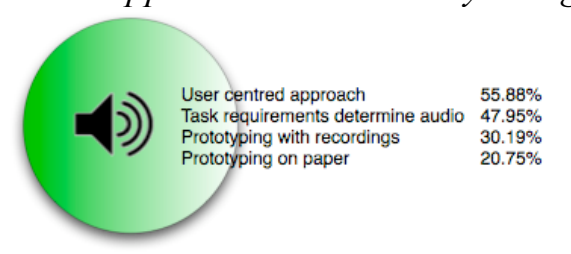

Asked for the initial steps in a design process targeting audio only design problems, over half of the participants stressed the importance of a user centred design approach (55.88\%). Around one third said they would investigate the context of use $(33.82 \%)$ and $19.12 \%$ stated task analysis. Less than one out of ten mentioned explicitly to conduct literature review or seek for guidance in publications $(8.82 \%)$. Other approaches mentioned were use cases, design space specification and (rapid) prototyping. For some $4.41 \%$ commercial implications were also important to be considered from the start. Interestingly, only $23.54 \%$ mentioned sound and $5.88 \%$ were concerned about sound mappings at this early stage. Also, 4 in 68 questioned the requirement for audio only (e.g., P24: "What made this a requirement when displays are easily available in most contexts").

Almost half of the participants said that user requirements are a main factor to determine the sound design $(47.95 \%)$, followed by the context of use $(35.62 \%)$ and the purpose of the application $(32.88 \%)$. Other factors mentioned were the properties of the content to be communicated, any technical constraints, the task requirements and usability (all between $10 \%$ and $17 \%$ ). Only $4.11 \%$ mentioned aesthetics as a main factor for the sound design.

Asked for techniques to create initial prototypes, $30.19 \%$ stated recording, $22.64 \%$ synthesis software and $20.75 \%$ the use of sound editing or sound mixing software. Only $9.43 \%$ mentioned sound libraries in this context; one mentioned Foley ${ }^{3}$. Interestingly, every fifth also mentioned work on paper like drawing or writing $(20.75 \%$, e.g., P51: "Initially in paper so that users and designer can imagine what is the real scenario". For $18.87 \%$ a "wizard of $\mathrm{oz}^{, 4}$ experiment would be their method of choice. Web designers naturally tended to use sound embedded in HTML markup and Flash $(11.32 \%$ and $9.43 \%)$. Four out of 53 (or $7.55 \%$ ) said they had no idea how they would create a prototype at all (e.g. P10: "Not really any idea, just include the sound in the prototype?").

On the question of how to include the user in the design process two-thirds $(65.28 \%)$ mentioned user/usability testing or more specifically listening/hearing tests $(15.28 \%)$. One third mentioned some form of participatory design (31.94\%) and $12.50 \%$ stated the users' involvement in specifying the requirements. Methodologically, (contextual) interviews were mentioned $(15.28 \%)$, as well as observation, quizzes, focus groups, wizard of oz experiments and cognitive task analysis.

\subsubsection{The Mp3 Player Example}

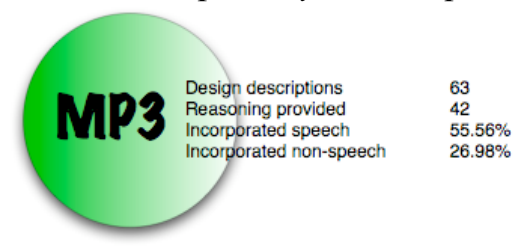

Given the task to think about the design of an Mp3-player with auditory feedback only, 63 participants provided quite extensive descriptions of their initial ideas; in average over 62 words per answer. The most striking fact is that over half of all solutions incorporated speech (55.56\%), while only $26.98 \%$ mentioned non-speech sounds. A considerable proportion of answers were mainly concerned about the button layout $(22.22 \%)$; e.g. which button would trigger which events. Two solutions incorporated gesture input, every tenth had speech recognition $(11.11 \%)$. With non-speech sounds, auditory icons were mentioned most often (4 times in 63 answers) followed by earcons ( 3 times) and spearcons (once). The use of spatial sound was suggested three times.

Three participants suggested to leave out the non-musical features that were requested in the task (e.g., P71: "I would kill the calendar and contacts"). Two participants said they do not know Mp3-players and gave no descriptions (P62: "Don't know - not familiar with Mp3-players - I must be old!”).

Forty-two participants provided their reasoning behind their design decisions. The majority stated that it was the first that came to mind (12 out of 42 or $28.57 \%$, e.g., P13: "Just seemed the obvious one...". Slightly less $(26.19 \%)$ stated usability related properties like speed, efficiency, simplicity, erroneousness, learnability and intuitiveness. Every tenth said the decisions are based on her/his experience $(11.90 \%)$. Some participants said they used speech for accuracy $(11.90 \%)$, while reasoning for non-speech sound varied from efficiency for additional feedback to the learning curve involved. Four out of 42 mentioned that their solution was a natural 'mapping'.

\footnotetext{
${ }^{3}$ Named after Jack Foley, Foley artists create natural sound effects for movies such as footsteps and horses' hooves.

${ }^{4}$ A method for HCI experiments in which subjects interact with a computer system that the subjects believe is autonomous, but is operated by a human being.
} 


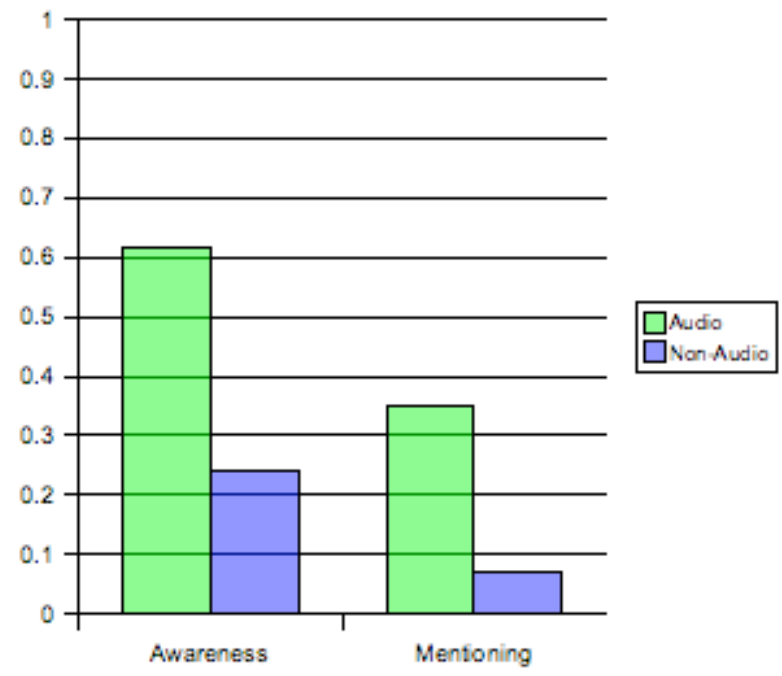

Figure 2. Awareness and mentioning of guidelines in the audio and non-audio groups.

Only 4 mentioned that they were exploiting standards, notably one said, "I can visualise it..." (P11).

\subsubsection{Guidance and Tools}

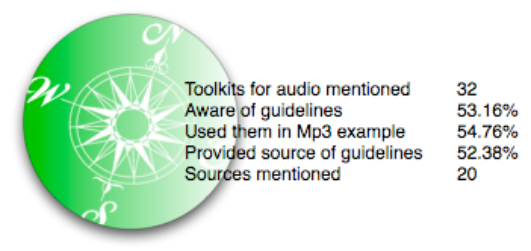

Slightly more than half of all participants provided some answer to the question about techniques, programming languages and toolkits to realise audio. Eleven, however, stated they did not know any, which makes it $41.86 \%$ or 36 out of 86 . Thirty-two different toolkits and languages were mentioned of which the following were the most frequently stated: $\mathrm{C}$ and $\mathrm{C}++$ $(27.78 \%)$, Pure Data (25\%), CSound (25\%), SuperCollider (22.22\%), Max/MSP and ChucK (19.44\% each). Also 13.89\% claimed they knew several or most of the available tools.

Over half of the participants said they were aware of some guidelines or principles for the design of auditory displays $(53.16 \%)$. In the group of participants who have not used audio in any of their designs this drops to $24.14 \%$. On the other hand, only $61.4 \%$ who used audio claimed to be aware of guidelines ${ }^{5}$.
When asked to provide the source of these guidelines or principles only every second of those who claimed to be aware of some did so $(52.38 \%)$. Twenty different sources were mentioned of which the most popular were Stephen Brewster's guidelines for designing earcons [5] (40.91\%), Stephen Barrass' auditory design space [6] (31.82\%), Gregory Kramer's book on auditory displays [7] (13.64\%) and Bill Gaver's and Meera Blattner's work on auditory icons and earcons $[8,9](9.09 \%$ each). Two out of 22 claimed to know several guidelines without specifying which. Again there is a significant difference between participants who did use audio in their design and these who did not: only $6.90 \%$ of those who have not used audio mentioned guidelines while at least a third of those who had some prior experience with audio did provide some $(35.09 \%)$. Figure 2 illustrates the numbers.

Half of the participants who were aware of some guidelines said they were influenced by them when developing the design of the Mp3-player example (54.76\%), while almost three-quarters said they used them in other designs $(73.81 \%)$. About half of the participants who provided a solution for the Mp3-player said they used other guidelines or principles, not specifically aimed at auditory display (51.85\%). Generic guidelines for user interfaces (6 times), usability ( 5 times), user centred design (3 times) and accessibility (twice in 20 answers) were mentioned most often. The majority said they were influenced to a great extent by those guidelines $(58.33 \%$ either great or very great extent), while $25 \%$ said to a moderate extent, $16.67 \%$ to a slight extent and none said not at all.

\subsubsection{Final Comments}

In general, most participants believed that audio can improve human-computer interaction. Roughly a third said to a very great extent $(32.93 \%), 25.61 \%$ to a great extent, $35.37 \%$ to a moderate extent and only $4.88 \%$ to a slight extent. Only one participant said not at all. Interestingly, there is only a slight shift between the participants who used audio and those who did not regarding this question: while $48.28 \%$ from the former group said audio can improve HCI to a great or very great extent, $66.57 \%$ believed this in the later group. The question on which guidance would participants like to be available for auditory displays provoked a great variety of answers which could not be as easily categorised. This and other particular interesting answers are subject of further analysis by qualitative text analysis in the subsequent section.

Table 1 summarises selected data and compares answers of participants who have designed audio in the interface before, with designers who have not.

Table 1. Comparison of answers of designers who have not used audio in the user interface before and designers who hav

\begin{tabular}{|lllll|}
\hline Probed information & Non-audio & \% in group & Audio & \% in group \\
\hline Number of participants & 29 & $100 \%$ & 57 & $100 \%$ \\
\hline Aware of audio related guidelines & 7 & $24.14 \%$ & 35 & $61.40 \%$ \\
\hline Mention audio related guidelines & 2 & $6.90 \%$ & 20 & $35.09 \%$ \\
\hline Mention "user centred design" in initial design approach & 13 & $44.83 \%$ & 25 & $43.86 \%$ \\
\hline Mention "context of use" in initial design approach & 9 & $31.03 \%$ & 14 & $24.56 \%$ \\
\hline Mention speech in the Mp3 example & 11 & $37.93 \%$ & 24 & $42.11 \%$ \\
\hline Mention non-speech in the Mp3 example & 5 & $17.24 \%$ & 12 & $21.05 \%$ \\
\hline Provide reasoning for the design of Mp3 player example & 14 & $48.28 \%$ & 28 & $49.12 \%$ \\
\hline Audio can improve HCI to a (very) great extent & 14 & $48.28 \%$ & 38 & $66.67 \%$ \\
\hline
\end{tabular}

\footnotetext{
${ }^{5}$ The discrepancy in the overall sum of percentages was caused by the fact that some participants did not answer both questions.
} 


\subsection{Qualitative Analysis}

The approach we chose to analyse the data from a qualitative perspective is based on methods used in ethnographical studies of text. Using a software tool to markup passages in texts we identified themes that were inherently conveyed. The TAMS Analyzer ${ }^{6}$ allowed us to scan the responses conveniently and mark up phrases of significance with different tags and also provided powerful search and sorting features to analyse aspects in the data. As in our quantitative analysis, we did not define the tags in advance, but let them emerge as we worked on the texts. The method was to read the answers with each research question in mind and find themes that would either support or counter assumptions.

\subsubsection{The Role of Audio in HCI}

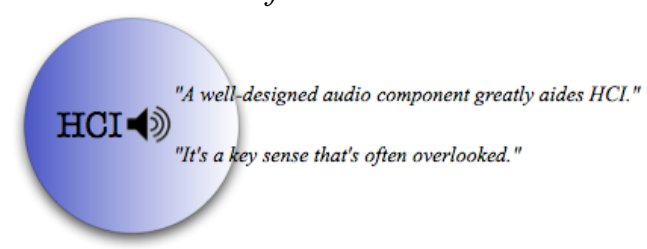

Auditory display design is clearly perceived as part of HCI in general. Almost every participant made references to generic HCI methods that are already established in HCI during the early stages of an auditory design. One stated, "I don't see that there's a difference here compared to ordinary design projects". (P73) or "Basic design is the same regardless of the specific UI" (P23). Looking at the results from the quantitative text analysis above confirms this: task analysis, participatory design and context of use are all well established concepts in the field of human-computer interaction.

Participants also suggest auditory display design being tightly integrated into a holistic, multi-modal approach - P69: " $A$ balanced design, using multiple senses and mental processes, allows for a wide range of effective and efficient interaction solutions". Some people made more general statements like "It's a key sense that's often overlooked" (P45) or "A welldesigned audio component greatly aides HCI" (P66). Other senses, however, are overlooked too - "(Audio) It's 50\% of your experience (if one counts visual as the other 50\%)" (P50).

\subsubsection{The Attitude Towards Audio}

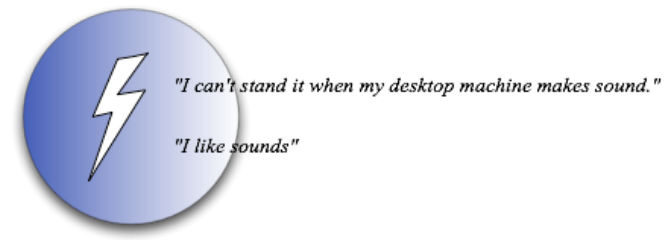

Many participants stated scepticism about the use of audio in human-computer interaction and doubted its efficiency. This was expressed in many different ways, some simply disbelieved in the capabilities of audio without providing any reason - P19: "I'm not confident that any audio solution would make the system really usable". Others explicitly listed issues with the auditory interaction channel, mostly regarding privacy and annoyance - P32: "Verbal is less private than visual and interacts more with people's surroundings", and P24: "Have to be careful -- could be VERY annoying". Another participant stated "Due to its intrusive nature, I see audio solutions as

${ }^{6}$ Text Analysis Markup System Analyzer

http://tamsys.sourceforge.net more valuable for expert users than novices" (P15), which is interesting as it positions audio as a mode that demands high skills, suggesting it needs expertise to be able to cope with it. Furthermore, it implies that audio cues are less intuitive. Another issue mentioned was cognitive load - P19: "The burden on the user's memory has seemed to me to be a significant barrier to ease of use".

In many cases participants had prejudices - P70: "Audio output is a very low-bandwidth way to communicate", or P36: "Untrained people don't like 'musical interfaces",. Some participants were concerned about synthesised audio - P43: "A lot of synthesised audio still has a rather low quality" - and stressed the importance to create audio that mimics reality P84: "They need to be realistic and match the real world as much as possible". Another interesting point of view was taken by three participants who all stated that "...input and output modality should be the same" (P24).

Also, the way participants expressed their scepticism was interesting to analyse and ranged from cool and focused statements to more emotional answers. Statements like "I don't want applications introducing auditory 'noise' into my environment. I want CONTROL over audible output" (P63), "I can't stand it when my desktop machine makes sound" (P70) and "Off by default" (P22) show that some participants, as users, have had bad experiences with audio in interfaces.

We also detected emotional resistance against designing audioonly interfaces. Asked for the Mp3-player design P16 answered simply: "Put a screen on it!" or as P30 put it: "..no normal sighted users are expected to accept this player!". Participants also questioned the task itself - P52: "But I would seriously question why the client had decided not to have a screen" and she/he went on "Perhaps show them some examples of how unusable this can be", implying her/his doubts about the success of such a design. P62 expressed his resignation: "Audio only interfaces have so many issues".

Besides all this negative feedback, participants also expressed their believe in auditory display design as a valuable contribution to human-computer interaction. The way they expressed themselves varied and ranged from listing advantageous features to simple statement such as "I like sounds" (P35). One participant said, "As compared to other forms of continuous feedback in artefacts, audio is inexpensive!" (P86); another stated "User security encouraged us to prefer auditory interfaces over graphic ones for mobile apps" (P27). But no one else outlined more advantageous properties of the auditory channel.

\subsubsection{Approach to Audio-Only Design}

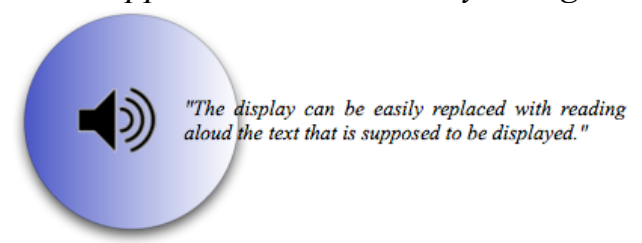

Asked to provide their initial ideas to design an auditory display for an Mp3-player the majority of them described their ideas quite extensively (see above). Notably, one participant expressed her/his discomfort with the question - "The expectation that designers should provide an instant solution is one of the reasons so many auditory interface are badly designed", and goes on "I would base my design on a structured analysis rather than the first idea" (P37). This is 
certainly valid for many design disciplines, but might be specifically true in the auditory domain.

Others, who provided their first ideas, gave surprisingly conservative accounts; despite the explicit encouragement to think out of the box and not to consider technical feasibility. A possible explanation is that many participants associated with auditory displays the use of speech only - "The display can be easily replaced with reading aloud the text that is supposed to be displayed" (P18). Another reason might be the unawareness of what is technically possible with audio and sound. This is supported by the fact that more innovative technologies like spatial audio were mentioned very little.

A related issue with auditory displays seems to be the problem of creating prototypes during the design process. Statements like "Ooh, no idea... doing the equivalent of paper design ... but with sound" (P12) show that a well known and easily accessible (as paper is without a doubt) way of creating prototypes is missing in this design discipline.

\subsubsection{What Guidance?}

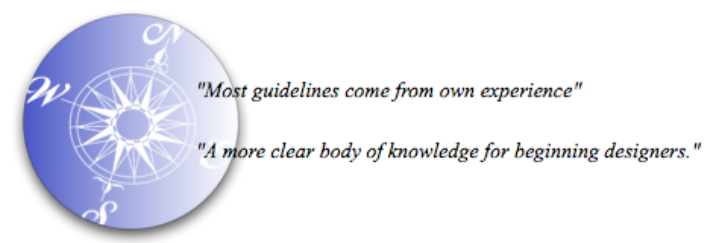

Asked for forms of guidance participants would like to see for auditory display design they provided a diversity of suggestions. Tested examples and approved standards were amongst the ones mentioned most often - "Just a set of examples (concise) that show what has been done before" (P80) or "Standard interactive controls" (P58). A main driving force behind these suggestions was expressed by P78: "Approved standard of audio use that could be applied without extensive user testing and research". Another aspect connected to the term 'standard' is provided by P55: "Successful strategies of tested/accepted designs". Standards are not only tested solutions, but also accepted by users.

Another related form of guidance incorporating examples are patterns, which were mentioned twice - "Design patterns are useful" (P86). One participant addressed the problem of the accessibility of examples - P59: "Given an interface problem, a similarity-rated list of inspiring successful interfaces for related situations". Many participants stressed the importance of the context of use when designing auditory displays - P67: "The appropriateness of using audio is very much dependent on the context of use", and P61: "Specific guidelines for different situations". P59 argues for using the context of use as an organising principle: "A well structured map of use cases / tasks / constraints \& real-world examples".

A more critical point of view about the value of examples is expressed by P37: "Auditory displays people have fewer examples to build upon". She/he goes on to say, "Until we have developed many successful auditory displays I believe we need to use design methods heavily based in theories of attention to develop new displays". An interesting statement in this context was made by P12: "I'm not $100 \%$ convinced that guidance has helped us in other areas". She/he goes on "Experience, and working with target audiences as much as possible, provides a much greater chance to do something that actually works". She/he stresses the value of experience in the design process and is supported by other participants - "Most guidelines come from own experience" (P59).
Two participants were concerned about the particularities of the target group, the designers themselves - P1: “...detailed and high level versions of the methodology to fit different levels of user expertise", and P13: "A more clear body of knowledge for beginning designers". In her/his attempt to explain further the differences between levels of guidance for different levels of expertise, she/he also makes a remarkable statement about experienced designers: "...but free the designer to use experience and creativity". In other words, while beginners may rely on more specific guidelines to create auditory displays, experts in the field appreciate their freedom in design choices to accommodate creativity and experience.

\subsubsection{Final Remarks}

Finally, this survey also provoked expressions of all kinds of attitudes, ranging from "Stop asking me silly questions, PLEASE" (P68 who nevertheless continued to fill out the questionnaire) to "You have successfully made me think more innovatively this morning :)" (P17). Either way, the 86 participants of this survey contributed greatly to a more detailed picture of the auditory design process, the needs of designers and the specific design problems with auditory displays.

\section{SUMMARY AND DISCUSSION}

The analysis revealed a number of valuable insights into the current practice of auditory display design. In this section we will summarise the findings and develop them into a set of requirements for a methodological framework for auditory display design.

\subsubsection{The Bigger Picture}

Strategies to approach design problems including audio vary. Many established high-level concepts in HCI may serve as a starting point. A methodological framework that supports designers to incorporate audio has therefore to fit seamlessly in the bigger picture and blend effortless with existing methods.

To make use of all human sensory capabilities for the interaction with technology is a high aim. A "balanced design using multiple senses", as P69 phrased it, requires a methodology that supports the design of interaction across modalities. The context of use is seen as a key property to inform the design and must play a prominent role in a holistic methodological framework.

The completeness of the auditory design space appears also to be an issue. As perceived by many HCI designers, this space consists almost exclusively of speech, not considering nonspeech sounds as powerful means to convey information. A framework must therefore make explicit the range of possibilities that the auditory interaction channel has to offer.

\subsubsection{Opinion Shaping}

Designers are also users, and many of them have had disappointing experiences with audio in the user interface. This resulted in prejudices and disbelieve in what audio can do for their designs. It seems to be a common pattern that audio is directly associated with annoyance and is not considered as a serious alternative to the visual channel. For a design framework this means it has to help designers overcome their initial scepticism and make designers re-consider using audio in the user interface. An obvious way of changing this public opinion would be the availability of a 'killer application'. The lack of such, however, could also be a result of common believe. 
Table 2: Summary of requirements for a framework to support the design of audio in the user interface.

\begin{tabular}{|l|l|}
\hline Domain & Requirement \\
\hline \multirow{3}{*}{ Bigger picture } & Blend in with established methods in HCI. \\
\cline { 2 - 2 } & Extendable for multi-modal designs. \\
\cline { 2 - 2 } & Conceptualise the design space to make designers aware of the range of possibilities. \\
\hline \multirow{2}{*}{ Opinion shaping } & Appealing to overcome prejudices and common disbelieve in audio. \\
\cline { 2 - 2 } & Incorporate aesthetics. \\
\hline \multirow{2}{*}{$\begin{array}{l}\text { Simplicity, } \begin{array}{l}\text { Experience and } \\
\text { Creativity }\end{array} \\
\text { Tools }\end{array}$} & Simple to use to provide a low entry barrier for novices and experts. \\
\cline { 2 - 2 } & Allow experts to incorporate their experience. \\
\cline { 2 - 2 } & Allow designers to exercise their creativity rather than being restricted by templates. \\
\cline { 2 - 2 } & Support the framework with design tools. \\
\hline
\end{tabular}

Sound can provoke strong emotional responses, which is both a strength and a weakness of the mode. Guidance to design sound for user interfaces has to incorporate a way of conveying good practice regarding aesthetics. An appropriate form of communicating such subtle values in designs is difficult to shape and will inevitably be source for debate.

\subsubsection{Simplicity, Experience and Creativity}

A successful design framework has to accommodate a variety of types of designers. The differences in the background of designers and their levels of expertise demand a flexible methodology that benefits novices and experts alike. While providing detailed guidance for designers new to the field, it must allow experts to incorporate their experience. This was rated as a key factor to good designs in the survey. The same is true for creativity. While novices may need to stick closer to what is suggested through the design framework, experts must be able to exercise their creativity without being restricted. Finally, the ease-of-use of the framework will be a major factor in its acceptance. Neither type of designer will accept highly theoretical approaches that demand substantial knowledge of the background to be able to create designs.

\subsubsection{Tools}

The majority of design tasks in HCI are supported by design tools at various levels. While paper and pen already constitute a powerful tool for designing graphical user interfaces, the lack or inaccessibility of tools in the auditory domain hinders the creation of prototypes or auditory sketches. A design framework must link its methods to powerful tools that allow the creation of external representations of ideas and concepts. Table 2 summarises the requirements we found to be key for the development of a framework for designing audio in the user interface.

\section{CONCLUSION AND FUTURE WORK}

In this paper we have presented a survey of common practice in auditory display design with the goal of laying the foundations for a methodological design framework for designing audio in the user interface. We provided the background and motivation of this research and described in detail the design of the survey and the analysis of the responses. Thanks to the high number of participants, we were able to draw an accurate picture of the current situation and developed the major insights into requirements for the design framework. These requirements are currently feeding directly into the development of such a framework using design patterns.

We envision a flexible and powerful framework that is implemented as an online information system, which guides designers through the process of incorporating audio in their designs. At its core the framework will organise design knowledge in the form of design patterns and examples according to their context. The 'context space' will function as a map to design problems and solutions. A more detailed description of these initial steps to develop this framework is provided in [10]. Future work will focus on evaluating the framework and developing the tools to support it.

\section{ACKNOWLEDGMENTS}

We would like to thank all participants who provided so rich and detailed responses and contributed greatly to a deeper understanding of the process.

\section{REFERENCES}

[1] Brewster, S. A. Overcoming the lack of screen space on mobile computers. Personal and Ubiquitous Computing, 6 (2002) 188-205.

[2] Nesbitt, K. V., and Barrass, S. Evaluation of a multimodal sonification and visualization of depth of market stock data. In Proceedings of the International conference for Auditory display (ICAD 2002). 2002, 233-239.

[3] Mynatt, E. D. Designing with auditory icons. In Proceedings of the international conference on Auditory display (ICAD 1994). 1994.

[4] Frauenberger, C., and Stockman, T. Patterns in auditory menu design. In Proceedings of the international conference on Auditory display (ICAD 2006). (London, UK, 2006). 2006, 141-147.

[5] Brewster, S. A. Providing a Structured Method for Integrating Non-Speech Audio into Human-Computer Interfaces. Ph.D. Thesis, University of York, UK, 1994.

[6] Barrass, S. Auditory Information Design. Ph.D. Thesis, The Australian National University, Australia, 1998.

[7] Kramer, G. (ed.), Auditory Display: Sonification, Audification, and Auditory Interfaces. Addison-Wesley, Reading, MA, 1994.

[8] Gaver, W. W. Using and creating auditory icons. In G. Kramer (ed.), Auditory display: Sonification, audification, and auditory interfaces. Addison-Wesley, Reading, MA, 1994, 417-447.

[9] Blattner, M. M., Sumikawa, D. A., and Greenberg, R. M. Earcons and icons: Their structure and common design principles. Human-Computer Interaction, 4 (1989), 11-44.

[10] Frauenberger C., and Stockman, T. Pacoad - Pattern design in the context space: A methodological framework for auditory display design. In Proceedings of the international conference on Auditory display (ICAD 2007). (Montreal, Canada, 2007). 2007. 
